\title{
Inverse Virtual Screening of Bradyoxetin and a Synthetic Intermediate using OOMD as a Source Database
}

\author{
Vanildo M. L. Braga \& Alex Taranto
}

\section{Introduction}

Quorum sensing bacteria produce and release chemical signal molecules (like $\mathrm{N}$-acyl homoserine, AHL1) that increase in concentration as a function of cell density. The responses cover a large spectrum of process such as the virulence in Staphylococcus aureus ${ }^{2}$, competence for DNA-uptake in Bacillus subtilis ${ }^{3}$ and Streptococcus pneumoniae, sporulation in Bacillus subtilis ${ }^{4}$, conjugal plasmid transfer in Enterococcus faecalis ${ }^{5}$, and bacteriocin production in lactic acid bacteria. The collapse of (AHL) signaling system in bacteria represents an attractive therapeutic approach towards the development of new antibiotics. Recently, a new extracellular modulator was isolated from a symbiotic bacterium (Bradyrhizobium japonicum)5 that nodulates soybean. This quorum sensing molecule, containing a novel oxetane ring, was partially characterized and named bradyoxetin (2-\{4-[[4-(3-aminooxetan-2-yl)phenyl](imino)methyl] phenyl oxetan-3-ylamine)(Figure 1). Since there's a lack of information (other than the nodulation of soy bean) it seemed extremely appropriate to investigate this unique organic scaffold using in silico techniques such as Inverse Virtual Screening (IVS) ${ }^{6}$ using our own molecular database (OOMD). IVS should provide us initial information on the biological profile of this molecule, as well as other synthetic intermediate, 3-azido-2-phenyl oxetane (Figure 1).



Figure 1. Structural formula and synthetic intermediate of 3-azido-2phenyl oxetane

\section{Experimental Procedures}

The molecules were entered using Marvin Sketch (ChemAxon, version 14.7.7). During this process the molecules were also protonated accordingly to its structure. The bradyoxetin series were able to accommodate three protons, one in the imine functionality, and other two on the amino groups attached to the oxetanes rings. The $\mathrm{pH}$ during protonation was set to be 7.4. The phenyl azido oxetanes were not protonated. During this process Geisteiger charges were also added. The ligand was set to be torsion free and the remaining structures were checked for incongruences in 3D. After this protocol the 
molecules had their energy minimized by MOPAC using $\mathrm{pm}^{7}$. The molecules were then opened in Auto Dock Tolls and were saved in pdbqt and in smiles for the purpose of expansion of a database of ligands.

The docking studies were performed using Vina1 0.27 . The data for the conf.txt files were extracted from the ligand present in the original pdb file (location of the biding site). Exhaustiveness was set to 8 and the grid box set to $20 \AA$ on each side.

With the docking finished, next step was to verify the graphical results. For this, we've used Discovery Studio v4.1.0.14169 (Accelerys Software Inc.).

Table 1 . The expressed results

\begin{tabular}{|c|c|c|c|c|c|c|c|c|c|}
\hline Mol. Target & $1 \mathrm{AGW}$ & 1DDX & $1 \mathrm{GKC}$ & $1 \mathrm{GMY}$ & 1LD8 & 1LF3 & 1LRH & 1QIB & 1QJA \\
\hline Lig. Crist. & -8.1 & -9.2 & -6.6 & -7.7 & -13.2 & -10.0 & -7.7 & -9.3 & -11.0 \\
\hline 5 & -5.6 & -7.5 & -6.9 & -5.6 & -5.6 & -5.9 & -6.2 & -6.8 & -5 \\
\hline 6 & -5.7 & -7.6 & -6.9 & -5.5 & -5.7 & -5.5 & -6 & -6.8 & -4.9 \\
\hline 7 & -5.6 & -7.8 & -7.2 & -5.8 & -5.6 & -5.4 & -6.5 & -7.1 & -5.5 \\
\hline 8 & -5.4 & -7.4 & -6.9 & -5.4 & -5.6 & -5.5 & -6.3 & -6.5 & -4.8 \\
\hline 1 & -7.8 & -9.3 & -9.4 & -7 & -7.8 & -7.6 & -4.9 & -9.3 & -6.2 \\
\hline 2 & -8.1 & -9.1 & -9.6 & -7.2 & -8.1 & -7.7 & -4.7 & -9.4 & -6.3 \\
\hline 3 & -8.2 & -9.4 & -9.5 & -7.1 & -8.2 & -7.7 & -4.9 & -9.4 & -6.4 \\
\hline 4 & -7.7 & -9.0 & -9.5 & -7.0 & -7.7 & -7.6 & -4.2 & -9.7 & -6.1 \\
\hline Mol. Target & 1W6M & 1W22 & $1 \mathrm{Z} 57$ & 1771 & 2ANL & $2 \mathrm{HYY}$ & $2 \mathrm{~K} 05$ & $2 \mathrm{QHN}$ & 2 VV9 \\
\hline Lig. Crist. & -4.3 & -9.0 & -9.5 & -7.6 & -10.0 & -12.6 & -33.3 & -7.4 & -9.1 \\
\hline 5 & -4.9 & -5.3 & -8.7 & -8.7 & -8.1 & -7.1 & -4.8 & -5.6 & -6.4 \\
\hline 6 & -4.6 & -5.2 & -8.8 & -8.6 & -7.7 & -7.2 & -4.7 & -5.7 & -6.5 \\
\hline 7 & -4.8 & -5.3 & -9.3 & -8.6 & -7.7 & -7.4 & -5.1 & -5.7 & -6.8 \\
\hline 8 & -5.0 & -5.3 & -8.8 & -8.7 & -8.2 & -7.0 & -4.7 & -5.6 & -6.3 \\
\hline 1 & -6.2 & -7.8 & -7.8 & -8.7 & -8.1 & -9.7 & -6.9 & -7.7 & -8.8 \\
\hline 2 & -6.2 & -8.1 & -8.1 & -8.6 & -7.7 & -10.2 & -7.2 & -7.7 & -8.8 \\
\hline 3 & -6.2 & -8.2 & -8.2 & -8.6 & -7.7 & -10.4 & -7.2 & -7.7 & -8.8 \\
\hline 4 & -6.2 & -7.7 & 0.0 & -8.7 & -8.1 & -10.0 & -6.7 & -7.7 & -9.1 \\
\hline Mol. Target & $2 \mathrm{YOE}$ & $2 Z O Q$ & 3BKY & 3BPF & $3 \mathrm{BZ3}$ & $3 \mathrm{C} 4 \mathrm{C}$ & $3 \mathrm{DV} 3$ & 3EDQ & 3ENE \\
\hline Lig. Crist. & -5.4 & -7.8 & -9.1 & -6.7 & -11.1 & -10.4 & -9.4 & -10.7 & -9.8 \\
\hline 5 & -5.7 & -6 & -5.5 & -4.9 & -6 & -6.2 & -5.6 & -5.2 & -6.1 \\
\hline 6 & -5.5 & -6.1 & -5.4 & -5.0 & -6.1 & -6.3 & -6.1 & -5.5 & -6.3 \\
\hline 7 & -5.8 & -6.3 & -6 & -5.1 & -6.1 & -6.6 & -6.2 & -5.8 & -6.5 \\
\hline 8 & -5.5 & -6.0 & -5.4 & -5.1 & -6.4 & -6.3 & -6.0 & -5.3 & -5.9 \\
\hline 1 & -8.1 & -8.2 & -7.1 & -6.9 & -8.2 & -9.3 & -9.1 & -7.3 & -8.2 \\
\hline 2 & -7.8 & -8.6 & -6.8 & -6.9 & -8.6 & -9.5 & -9.6 & -7.4 & -8.1 \\
\hline 3 & -8.0 & -8.6 & -7.3 & -6.8 & -8.7 & -9.8 & -9.6 & -7.4 & -8.0 \\
\hline 4 & -8.1 & -8.2 & -7.1 & -6.9 & -8.2 & -9.3 & -9.1 & -7.3 & -8.5 \\
\hline Mol. Target & 3FAP & 3FL5 & $3 \mathrm{G} 0 \mathrm{E}$ & $3 \mathrm{HIG}$ & $3 J Y A$ & $3 N 8 Z$ & $3 U_{11}$ & $4 A G N$ & 4EY7 \\
\hline Lig. Crist. & -19.4 & -11.5 & -10.4 & -9.1 & -8.4 & -9.3 & -9.6 ou $-7 . !$ & -6.5 & -12.7 \\
\hline 5 & -6.8 & -7.7 & -6.3 & -7.1 & -6.1 & -6.4 & -4.9 & -4.8 & -7.4 \\
\hline 6 & -6.4 & -7.2 & -6.2 & -7.1 & -6.2 & -6.1 & -5.6 & -4.9 & -7.3 \\
\hline 7 & -6.6 & -8.0 & -6.5 & -6.6 & -6.9 & -6.4 & -5.8 & -5.1 & -7.8 \\
\hline 8 & -6.9 & -7.5 & -6.2 & -5.5 & -6.2 & -5.8 & -5.3 & -6.5 & -7.2 \\
\hline 1 & -9.1 & -6.9 & -8.9 & -10.2 & -8.2 & -5.9 & -7 & -6.6 & -10.1 \\
\hline 2 & -9.4 & -6.8 & -8.7 & -9.9 & -8.2 & -5.6 & -7.3 & -6.4 & -10.4 \\
\hline 3 & -9.3 & -7.0 & -8.6 & -9.9 & -8.2 & -5.9 & -7.3 & -6.1 & -10.6 \\
\hline 4 & -9.2 & -7.5 & -9.1 & -9.8 & -8.2 & -5.9 & -6.9 & -6.5 & -10.2 \\
\hline Mol. Target & PFATP & PFHT & & & & & & & \\
\hline Lig. Crist. & -8.5 & -5.4 & & & & & & & \\
\hline 5 & -6 & -5 & & Legenda: & & & & & \\
\hline 6 & -6.0 & -5.7 & & & & & & & \\
\hline 7 & -6.1 & -5.7 & & cancer & & ativo & & & \\
\hline 8 & -6.0 & -5 & & dengue & & inativo & & & \\
\hline 1 & -8.5 & -7.4 & & malária & & & & & \\
\hline 2 & -8.3 & -7.8 & & & & & & & \\
\hline 3 & -8.3 & -7.7 & & & & & & & \\
\hline 4 & -8.5 & -7.4 & & & & & & & \\
\hline
\end{tabular}




\section{Results and Discussion}

The most significant results were found in $1 \mathrm{GKC} 8$, 1W6M9, 2YOE10. In these cases the difference between the natural complex (ligand plus receptor) and the created complex generated through docking was more expressive. In most cases, the targets are involved with cancer. $1 \mathrm{GKC}$ is part of the Matrix metalloproteinases (MMPs), the class of enzymes involved in the degradation of extra cellular matrix and surrounding cells are known to be expressed during cancer cell invasion, arthritis and metastasis and MMP-9, in particular seems to be a key protease associated with tumor progression. 1W6M is equally involved with cancer although through a different mode of action. It's presence is associated with cell migration and with tumor malignancy. $2 \mathrm{YOE}$ is a GABA receptor modulated by benzodiazepines.

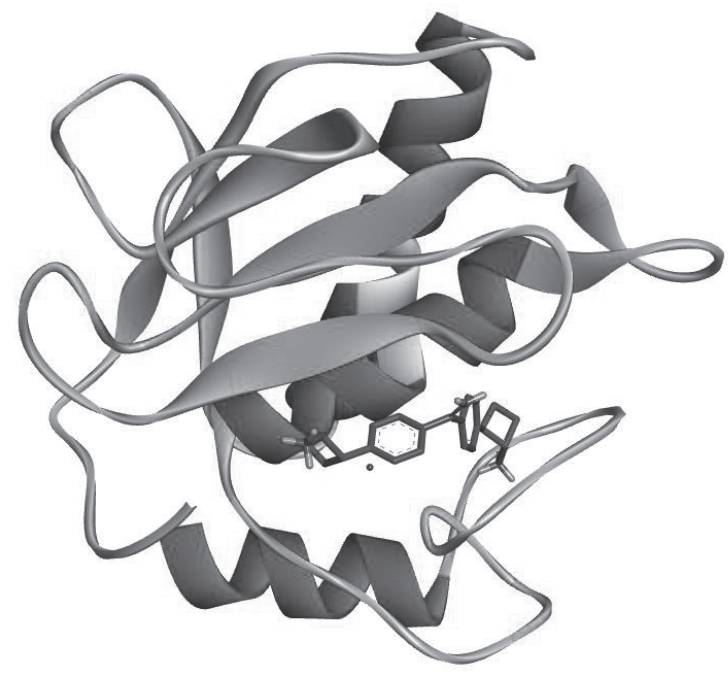

Figure 2. ligand 2 complexed with GKC

As it can be seen, looking to the pharmacophore map on figure 3, Pi interactions play a major role in binding process of ligand 2. In one of the rings there is a Pi stack created by a $\mathrm{Zn}$ atom and His 401 . The zinc atom also interacts with the second ring increasing the stability of the complex. The amino groups also play a pivotal role having also Pi interactions with Phe 110, His 405 and Tyr 423. The imine bridge also interacts with His 401 contributing for the low energy of the complex.

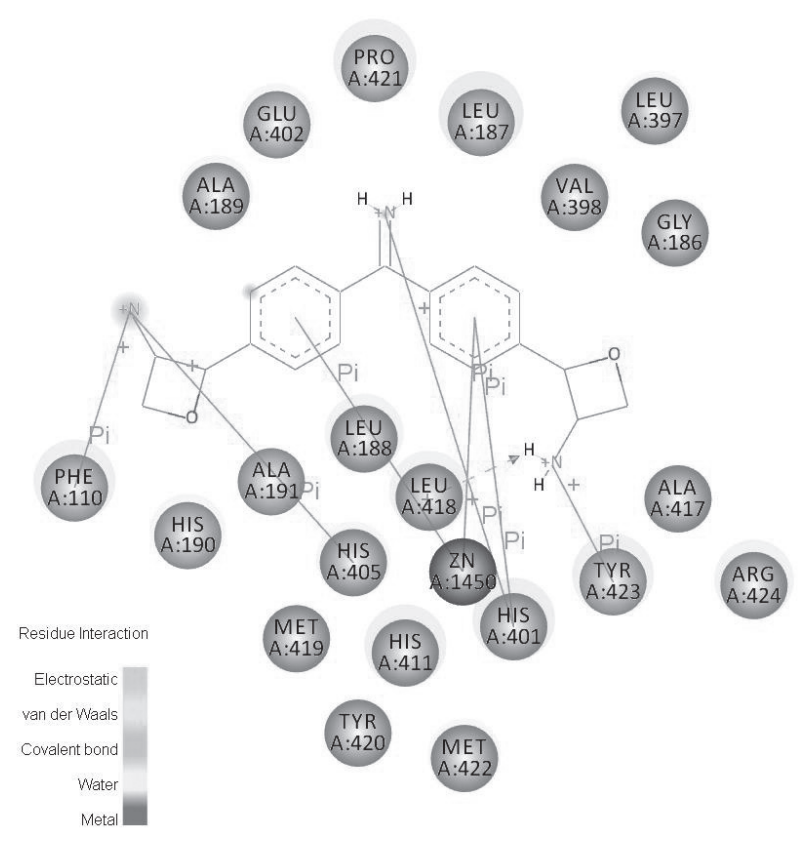

Figure 3. Pharmacophoric map of GKC complexed with ligand 2.

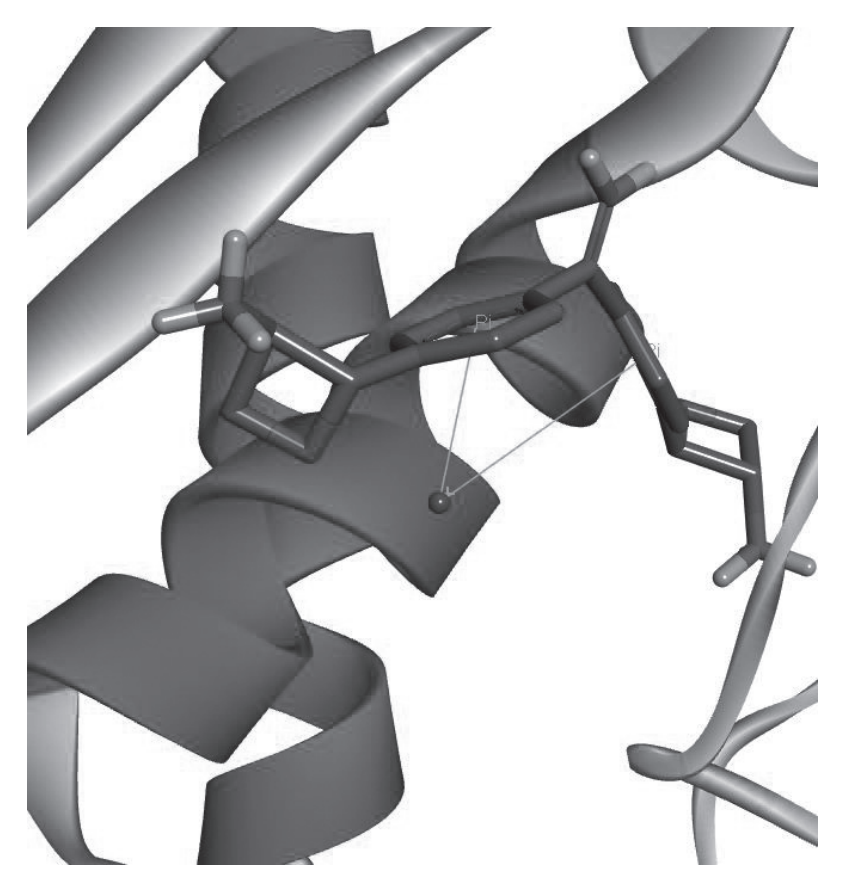

Figure 4. $\mathrm{Pi}$ interactions between $\mathrm{Zn}$ and aromatic rings of ligand 2 


\section{Artigo Geral 56}

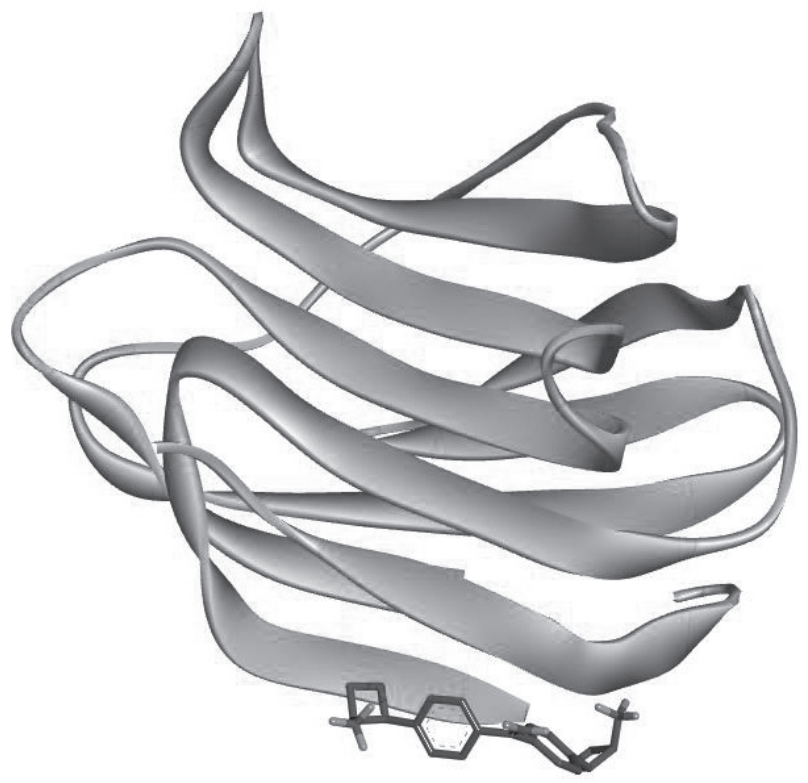

Figure 4. ligand 1 complexed with $1 \mathrm{~W} 6 \mathrm{M}$
As it can be seen in the figure above the number of interactions is much smaller than the previous example. Even so, it is enough to have a better value than the crystallographic complex. The key interactions are a Pi interaction between Phe 1133 and the imine moiety and hydrogen bonds between one of the amine groups and Glu 1015 and Asn 1010.

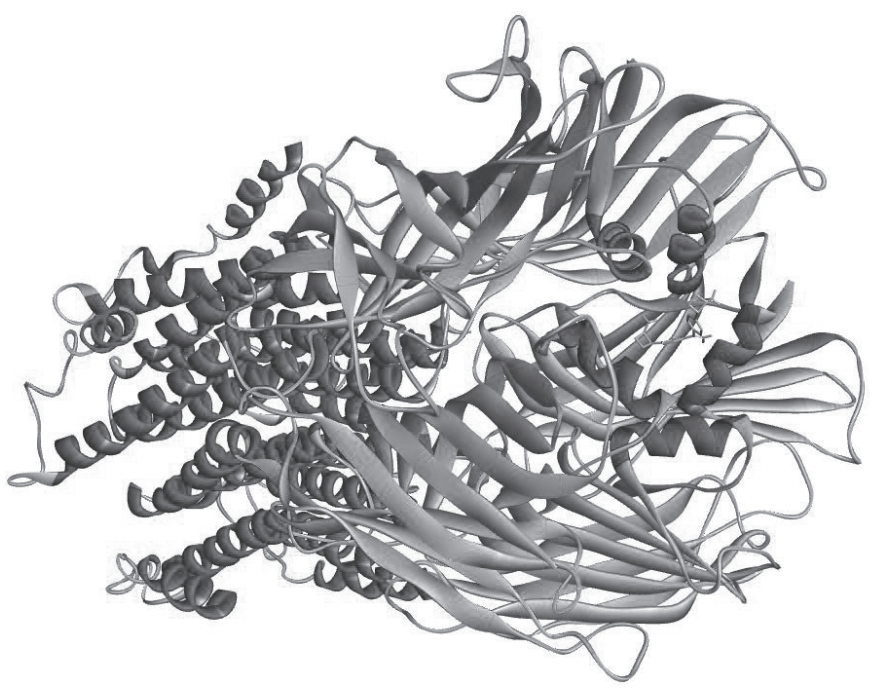

Figure 6. ligand 4 complexed with 2 YOE. The ligand is highlighted in yellow.

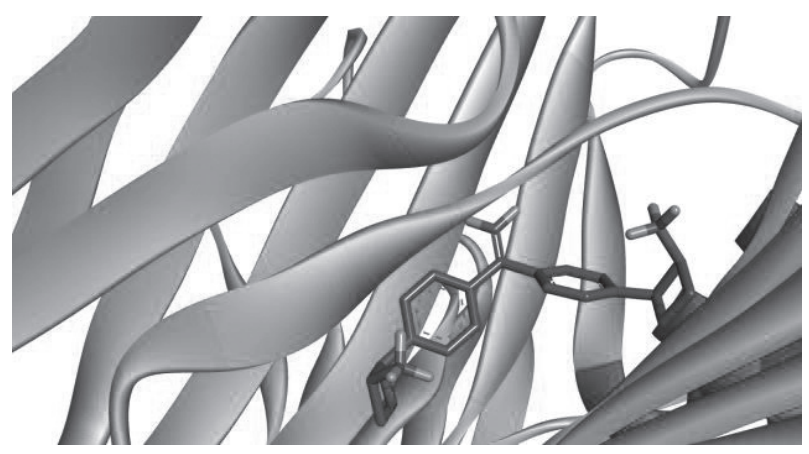

Figure7. A zoom taken off from Figure 6 
Phe 78 and Tyr 102 interact with 2YOE through Pi bonds with the aromatic ring and with the imine. Other interactions seen before are the hydrogen bonds between the protonated amines and the surrounding residues such as Val73, Glu59 and Leu 76.

\section{Conclusions}

The sources for therapeutic molecules seem to be infinite. What were the odds of a symbiotic bacterium that helps nodulation and therefore with nitrogen fixation on soy display good results in an in silico screening against cancer mainly. Although the results look promising, a biological study is mandatory to validate the in silico results.

The next step should be a refinement docking followed by molecular dynamics.

\section{Acknowledgments}

The authors are grateful for the support given from the FAPEMIG, CAPES and CNPQ.

\section{References}

1. Parsek, M. R. and E. P. Greenberg (2000). “Acyl-homoserine lactone quorum sensing in Gram-negative bacteria: A signaling mechanism involved in associations with higher organisms." PNAS 97(16): 8789-8793.

2. Johnson, Jeffrey G.; Wang, Boyuan; Debelouchina, Galia T.; Novick, Richard P.; Muir, Tom W. "Increasing AIP Macrocycle Size Reveals Key Features of agr Activation in Staphylococcus aureus" ChemBioChem (2015), 16(7), 1093-1100

3. Vidal, Jorge E.; Ludewick, Herbert P.; Kunkel, Rebekah M.; Zahner, Dorothea; Klugman, Keith P. "The LuxS-dependent quorum sensing system regulates early biofilm formation by Streptococcus pneumoniae strain D39" Infection and Immunity (2011), 79(10), 4050-4060.

4. Higgins, Douglas; Dworkin, Jonathan." Recent progress in Bacillus subtilis sporulation", FEMS Microbiology Reviews (2012), 36(1), 131-148.

5. Dunny, Gary M. "Enterococcal sex pheromones: signaling, social behavior, and evolution." Annual Review of Genetics (2013), 47, 457-482.

6. Loh, John; Carlson, Russell W.; York, William S.; Stacey, Gary. "Bradyoxetin, a unique chemical signal involved in symbiotic gene regulation." Proceedings of the National Academy of Sciences of the United States of America (2002), 99(22), 1444614451 .
7. Schomburg, Karen T.; Bietz, Stefan; Briem, Hans; Henzler, Angela M.; Urbaczek, Sascha; Rarey, Matthias. "Facing the Challenges of Structure-Based Target Prediction by Inverse Virtual Screening." Journal of Chemical Information and Modeling (2014), 54(6), 1676-1686.

8. Polikanov, Y.S., Melnikov, S.V., Soll, D., Steitz, T.A. "Structural insights into the role of rRNA modifications in protein synthesis and ribosome assembly." (2015) Nat.Struct.Mol.Biol. 22: 342344 .

9. Lopez-Lucendo, M.I.F., Solis, D., Andre, S., Hirabayashi, J., Kasai, K., Kaltner, H., Gabius, H.J., Romero, A. " Structural insights into the role of rRNA modifications in protein synthesis and ribosome assembly." (2015) Nat.Struct.Mol.Biol. 22: 342344.

10. Spurny, R., Ramerstorfer, J., Price, K., Brams, M., Ernst, M., Nury, H., Verheij, M., Legrand, P., Bertrand, D., Bertrand, S., Dougherty, D.A., De Esch, I.J.P., Corringer, P., Sieghart, W., Lummis, S.C.R., Ulens, C. "Pentameric ligand-gated ion channel ELIC is activated by GABA and modulated by benzodiazepines." (2012) Proc. Natl. Acad. Sci. USA 109: E3028.

\section{Vanildo M. L. Braga \& Alex Taranto}

Universidade Federal de São João Del Rei - Centro-Oeste campus 35501-296, Chanadour, Divinópolis-MG. 